\title{
When lack of practice helps: Retrieval following single presentations of categorized lists
}

\author{
DAVID BURROWS \\ Skidmore College, Saratoga Springs, New York
}

\begin{abstract}
Subjects were given a single presentation of a list of 8,12 , or 16 words, taken from one conceptual category. After attempting to recall the list words, they were given a reaction time retrieval test. Positive probes were taken from the list, and negative probes were taken from outside the list category. Reaction time did not differ with the number of memory list words for either type of probe. This is in contrast with results of previous experiments, which stressed careful memorization of the memory list and found increases in reaction time with number of memory words. The results suggest a holistic retrieval process.
\end{abstract}

One of the most important aspects of memory retrieval is that it takes time; the more information a person has memorized, the longer it takes that person to retrieve any particular memory item. This paper is concerned with ways in which time to retrieve can be shortened.

Sternberg (1966) demonstrated the basic retrieval time phenomenon in a recognition task that required subjects to indicate whether or not a test item had been present in a recently memorized list. Reaction time for the decision was an increasing linear function of the number of list items. This phenomenon is widely known as the setsize effect. The effect is often interpreted as evidence of an item-by-item search process, and the slope of the function represents the rate of that process. There have been several attempts to introduce variables that will eliminate or at least attenuate the effect, perhaps as a way of demonstrating that such variables will increase retrieval efficiency. Although some studies have shown that the setsize effect can be attenuated, the effect is robust and never seems to be completely abolished. Ross (1970) and Kristofferson (1972a) both showed that extended practice on the memory set will not abolish the effect, although some specific forms of practice can attenuate it (Kristofferson, 1972b). Naus, Glucksberg, and Ornstein (1972), Homa (1973), and Okada and Burrows (1973) have shown that organizing the memory list into identifiable sublists can reduce the set-size effect, particularly if a warning cue specifies that a particular sublist is "relevant." This is often interpreted as evidence for a selective search process in which retrieval is focused on a relevant sublist.

The present research looks at another way in which the set-size function can be attenuated. If the memory list can be converted into an alternative, unified representation, perhaps this representation can be checked in a single operation. If memory lists of different lengths can all be converted into such a unified representation, and each

The author's mailing address is: Department of Psychology, Skidmore College, Saratoga Springs, NY 12866-0851. checked in a single operation, the set-size effect would disappear. Such a result would imply a switch from the analytic item-by-item search to a holistic retrieval procedure. A situation that would seem to be a good candidate for creating a unified representation is one in which all of the memory items are words that belong to a single semantic category. If all test items are either list members (positive probes) or do not belong to the semantic category represented on the list (negative probes), then a single categorization test seems logically sufficient to make a decision and initiate a response. The critical test of this notion requires a condition in which memory list length varies, all list items belong to a single category, and negative probes are not drawn from the category of the memory set. A set-size effect with zero slope would indicate that decisions resulted from a single comparison between the test item and the entire memory list. Such a result would suggest the general possibility of efficient remembering through a single-operation retrieval of any set of information that is organized in some known fashion. Although the probable zero-slope result appears so likely that the experiment seems trivial, the available data do not support this holistic retrieval hypothesis. Homa (1973), Lively and Sanford (1972), and Milles and Morin (1970) included conditions in which there were negative probes not drawn from the category of the memory list and found set-size effects greater than zero. Burrows and Okada (1982) found such an effect even for subjects who were never tested with negatiave items drawn from the same category as the memory list, and who were encouraged to use this knowledge to make single-operation decisions.

One reason for the failure to obtain zero slopes may be that the above experiments used procedures that emphasized careful memorization of the individual list items. This might encourage subjects to rely upon individual item representations and to ignore the conceptual nature of the list. In the Burrows and Okada (1982) study, for example, subjects were not given the retrieval test until they had learned the individual items on the list with enough 
thoroughness to recall all list items perfectly on two consecutive trials. It is possible that this strict learning criterion focuses attention so heavily on the individual items that it leads to an item-checking strategy, even when this strategy is less efficient than a category-checking strategy. The present experiment tests this hypothesis by using single-category lists, negative probes taken from outside of the list category, and acquisition conditions that do not stress perfect recall of all list items.

\section{METHOD}

\section{Subjects}

The subjects were 18 undergraduates from an experimental psychology class at Skidmore College. The subjects were given extra credit in the course in exchange for participation. None of the subjects was informed of the hypotheses before the experiment, and none had previous experience with reaction time experiments.

\section{Apparatus}

The experiment was controlled by an Apple II + computer, equipped with an amber monitor. Stimulus words were presented on the monitor, and subjects signaled their responses by pressing keys on the computer's keyboard. The " $F$ "' key was used for a positive response, and the "J" key for a negative response. The computer was programmed to measure reaction times to the nearest millisecond.

\section{Procedure}

Each subject was tested on three lists, an 8-word list, a 12-word list, and a 16-word list. The order of the three tests was varied with a randomized block design. A test began with the presentation of the list words, one at a time. Each word was presented on the monitor for $2.5 \mathrm{sec}$, and was followed immediately by the next word. For any one list, each of the words belonged to a single conceptual category, drawn from the Battig and Montague (1969) norms. Nine of the Battig and Montague categories were used, with the target category drawn randomly from the set of nine. Once the target category was established, the list items were drawn randomly from the set of the 24 most dominant exemplars of that category. After the last word on the list had been presented, a row of asterisks appeared. This was the signal to recall the list words by writing down as many of them as could be remembered. Lined answer sheets were used for the recall procedure. The subjects were instructed to write down the words in any order they wished. Each list was presented once. Following the list recall, the recognition trials began. On each trial, a row of asterisks appeared for $1.5 \mathrm{sec}$. As soon as the asterisks disappeared, a test word was presented. The test word remained on the screen until the subject responded by pressing either the " $F$ " or the " $\mathrm{J}$ " key. After an intertrial interval of $0.5 \mathrm{sec}$, a new trial began with a row of asterisks. The test sequence consisted of 16 positive trials and 16 negative trials, randomly ordered. On positive trials, the test word was randomly selected from the memory list. On negative trials, the test word was randomly selected from one of the eight category sets not used as the target category. After the 32 test trials, presentation and testing of the next list began. This continued until all three lists had been presented and tested. For any single subject, the three target lists were drawn from three separate conceptual categories.

The subjects were instructed to make a positive response on the test trials if the test word was a member of the previously presented word list, and to make a negative response otherwise. They were made aware of the fact that negative probes did not belong to the same category as words in the memory list, and were encouraged to use this information in accomplishing their task.

\section{RESULTS}

Table 1 shows the data for the recall task. The table shows number of recalled words as a function of set size, and proportion of words recalled as a function of set size.
As set size increased, the number of recalled words also appears to have increased, but the proportion of list words recalled decreased. An analysis of variance confirmed the increase in number of recalled words with increasing set size $[F(2,34)=51.3, p<.01]$. A separate analysis was performed on the proportion correct, after the application of an arcsin transformation. This analysis confirmed the decrease in proportion correct $[F(2,34)=13.5$, $p<.01]$.

Table 2 shows the accuracy data for the recognition task. Entries represent proportion of correct answers as a function of set size and type of response (positive/negative). Errors were almost nonexistent and do not seem to be related to either of the independent variables. A 3 $\times 2$ analysis of variance was performed on the data, after application of an arcsin transformation. None of the obtained $F$ values approached significance.

Table 3 shows the reaction time data for the recognition task. Entries are mean reaction times for correct responses, as a function of both set size and type of response. It is evident that reaction time did not increase with set size for either type of response. The best-fitting linear functions relating reaction time to set size $(s)$ are:

$$
\begin{aligned}
& \mathrm{RT}=-7.4 s+724 \text { (positive responses) } \\
& \mathrm{RT}=0.9 s+622 \text { (negative responses) } \\
& \mathrm{RT}=-4.6 s+690 \text { (all responses) }
\end{aligned}
$$

A $3 \times 2$ analysis of variance confirmed the absence of a set-size effect [for set size, $F(2,34)=1.26$, n.s.; for type of response, $F<1]$. There was a significant set size $\times$ response type interaction $[F(2,34)=3.27, p<.05]$.

Table 1

Mean Number and Proportion of Words Recalled as a Function of Set Size

\begin{tabular}{lccc}
\hline & \multicolumn{3}{c}{ Set Size } \\
\cline { 2 - 4 } & 8 & 12 & 16 \\
\hline Words Recalled & 7.50 & 10.27 & 12.33 \\
Proportion Recalled & 0.94 & 0.86 & 0.77 \\
\hline
\end{tabular}

Table 2

Mean Proportion Correct on Recognition Task as a Function of Set Size and Type of Response

\begin{tabular}{llllc}
\hline & \multicolumn{4}{c}{ Set Size } \\
\cline { 2 - 5 } Response Type & 8 & 12 & 16 & All \\
\hline Positive & .97 & .97 & .99 & .98 \\
Negative & .97 & .97 & .98 & .97 \\
Combined & .97 & .97 & .99 & \\
\hline
\end{tabular}

Table 3

Mean Reaction Time (in Milliseconds) for Correct Responses on Recognition Task as a Function of Set Size and Type of Response

\begin{tabular}{lcccc}
\hline & \multicolumn{4}{c}{ Set Size } \\
\cline { 2 - 5 } Response Type & 8 & 12 & 16 & All \\
\hline Positive & 691 & 608 & 610 & 637 \\
Negative & 627 & 636 & 634 & 632 \\
Combined & 659 & 622 & 622 & \\
\hline
\end{tabular}


Inspection of the data suggests that this interaction reflects a slower mean reaction time for positive responses with Set Size 8 and faster reaction times for positive responses at the other two set sizes.

Clearly the most striking aspect of the data is the virtual absence of any set-size effect, as revealed both by the slope constants that are close to zero and the lack of set-size effect in the analysis of variance.

\section{DISCUSSION}

The data clearly show the absence of a set-size effect. Reaction times are nearly constant across set sizes. This confirms the hypothesis that the subjects were able to make holistic comparisons between the test item and the memory information when engaged in the retrieval task. The most obvious interpretation is that the subjects made category judgments of the test items, rather than comparing each test item with each word in the memory set. The absolute reaction time values are consistent with this interpretation; they are lower than usually found in memory search tasks with comparable list lengths, and similar to those found in categorization judgments.

It is important to note that accuracy of performance was high, both on the recognition task and on the recall task. The recall data show that the subjects actually made an effort to encode and memorize the word sets. If this were not the case, the reaction time data could be interpreted as showing that subjects simply ignored the memory words, and approached the recognition task by engaging in a categorization judgment that is meaningless in terms of retrieval of particular memory items. The increase in number of words recalled with set size, combined with the generally high level of recall accuracy, removes some of the force of this interpretation. The high accuracy in the recognition task is also considered important in tasks of this type; permitting subjects to make many errors would open the possibility that some unknown subject strategies are being used.

The subjects were not given any final recall test, and it is possible that they ignored the actual memory words once the recognition task began. The lack of a final recall test is common in experiments similar to this one. In similar experiments that did show increases in reaction time with set size (despite the absence of intracategory negative probes), intensive testing of learning during the acquisition phase seemed sufficient to create a set-size effect during the recognition testing phase.

Finally, the results are ironic but instructive; it is possible that spending time in the rote learning of information may hinder retrieval. Perhaps it is true that we should pay attention to the forest and not concentrate too much on the trees.

\section{REFERENCES}

Battig, W. F., \& Montague, W. E. (1969). Category norms for verbal items in 56 categories: A replication and extension of the Connecticut category norms. Journal of Experimental Psychology Monograph, 80(3, Pt. 2).

BurRows, D., \& OKADA, R. (1982). Retrieval of item and category information: Evidence for an interactive model. Canadian Journal of Psychology, 36, 655-669.

Homa, D. (1973). Organization and long-term memory search. Memory \& Cognition, 1, 369-379.

KRISTOFFERSON, M. W. (1972a). Effects of practice on characterclassification performance. Canadian Journal of Psychology, 26, 540-560.

KrISTOFFerson, M. W. (1972b). When item recognition and visual search functions are similar. Perception \& Psychophysics, 12, 379-384.

LIVELY, B. L., \& SANFORD, B. J. (1972). The use of category information in a memory search task. Journal of Experimental Psychology, 93, 379-385.

Milles, K. P., \& Morin, R. E. (1970, May). The effect of a basis for stimulus classification on recognition reaction time. Paper presented at a meeting of the Midwestern Psychological Association, Cincinnati.

Naus, M. J., Glucksberg, S., \& OrnsteIn, P. A. (1972). Taxonomic word categories and memory search. Cognitive Psychology, 3, 643-654.

OKADA, R., \& Burrows, D. (1973). Organizational factors in highspeed scanning. Journal of Experimental Psychology, 101, 77-81.

Ross, J. (1970). Extended practice with a single-character classification task. Perception \& Psychophysics, 8, 276-278.

STERNBERG, S. (1966). High-speed scanning in human memory. Science, 153, 652-654.

(Manuscript received for publication July 17, 1986.) 